\title{
Lipid-based nanoparticles for the therapeutic delivery of non-coding RNAs in breast cancer (Review)
}

\author{
MACRINA B. SILVA-CÁZARES ${ }^{1}$, MARÍA Z. SAAVEDRA-LEOS ${ }^{1}$, EUCLIDES JORDAN-ALEJANDRE ${ }^{2}$, \\ STEPHANIE I. NUÑEZ-OLVERA ${ }^{2}$, ISAAC CÓMPEAN-MARTÍNEZ ${ }^{1}$ and CÉSAR LÓPEZ-CAMARILLO ${ }^{2}$ \\ ${ }^{1}$ Institutional Doctorate in Engineering and Matherials Science, Autonomous University of San Luis Potosí, \\ San Luis Potosí $78760 ;{ }^{2}$ Oncogenomics Laboratory, Autonomous University of México City, México City 03100, México
}

Received February 23, 2020; Accepted August 18, 2020

DOI: $10.3892 /$ or.2020.7791

\begin{abstract}
Breast cancer is the most common type of cancer with the highest morbidity and mortality rates in women worldwide. Recent efforts to improve the current antitumor therapies have led to the development of novel treatment approaches based on the delivery of therapeutic non-coding RNAs (ncRNAs) using nanotechnology. Treatment methods using lipid-based nanoparticles (LBNPs) have greatly improved the delivery efficiency of ncRNAs into tumor cells and tissues. This type of delivery approach has provided significant advantages, such as reduced therapeutic doses, lower cytotoxicity to normal cells and the ability to reverse resistance to chemotherapy. LBNPs have demonstrated the ability to deliver therapeutic ncRNAs, more specifically microRNAs (miRNAs) and small interfering RNAs (siRNAs); this has been reported modulate the expression levels of oncogenes and tumor suppressor genes involved in several biological processes, including cell growth and proliferation, cell death, invasion and metastasis, thus impairing the malignant behavior of tumors. Therefore, ncRNA-based therapies combined with the LBNP delivery strategy, namely nanomiRNAs, may represent a promising antitumor strategy guaranteeing superior biocompatibility, higher biodegradability, lower immunogenicity and decreased toxicity to normal cells compared with other therapeutic approaches. The present review summarized the current knowledge of the application of LBNPs for delivering miRNAs and siRNAs in breast cancer cells and mouse models, in addition to discussing their promising antitumor effects.
\end{abstract}

Correspondence to: Dr César López-Camarillo, Oncogenomics Laboratory, Autonomous University of México City, San Lorenzo 290, México City 03100, México

E-mail: cesar.lopez@uacm.edu.mx

Key words: lipids, nanoparticles, small interfering RNAs, microRNAs, breast cancer

\author{
Contents \\ 1. Introduction \\ 2. Lipid-based NPs (LBNPs) for non-coding RNA (ncRNA) \\ delivery in breast cancer cells \\ 3. Cationic liposomes \\ 4. Neutral liposomes \\ 5. Ionizable liposomes \\ 6. Exosomes nanocarriers \\ 7. Stealth liposomes \\ 8. Triggered-release liposomes \\ 9. Porphysomes \\ 10. Lipid-coated calcium phosphate (LCP) NPs \\ 11. Conclusions
}

\section{Introduction}

Cancer is a multifactorial disease caused by genetic, epigenetic and environmental factors, and represents the end point of multiple molecular changes, which results in the dysregulation of the normal biological processes controlling cell proliferation, cell survival, genome stability, energy metabolism, angiogenesis and immune surveillance to promote tumorigenesis (1). Breast cancer is the most common type of cancer among women and the main cause of cancer-related deaths, accounting for 2.08 million new cases and 626,679 deaths worldwide annually (2). It has been estimated that the incidence of breast cancer will reach 3.2 million cases worldwide by 2050 (3). Breast cancer treatment is primarily focused on local intervention, including surgery and radiotherapy, while systemic treatments consist of chemotherapy, hormonal regimens, targeted-therapies and immunotherapy (4). Patient survival rates depend on the stage of disease, and the presence of metastases, as well as the existence of drugs resistance. Therefore, there is a urgent requirement for the implementation of novel and more effective therapies, such as the use of drugs-coupled nanoparticles (NP) that may efficiently target the tumors, which are currently under exhaustive investigation (5). To understand the most common phenotypic alterations that occurred in heterogeneous tumors, Hanahan and Weinberg (6) described the typical features of malignancies, known as the hallmarks of cancer, as the ability of cancer cells to: i) Exhibit 
sustained proliferative signaling; ii) evade growth suppressors; iii) resist programmed cell death; iv) enable replicative immortality; v) induce angiogenesis; and vi) activate invasion and metastasis. In 2011, Hanahan and Weinberg (7) proposed two additional cancer hallmarks, namely the reprogramming of energy metabolism and the ability to evade immune responses; the authors further suggested that both hallmarks were enabled by two traits, namely genome instability and mutations. Cancer is not considered as a single disease, therefore, breast tumors with different diagnostic features differ in the hallmarks controlling their clinical behavior and patient outcome. Personalized therapies are directed against proteins and signaling pathways governing cancer hallmarks, thus highlighting the importance of identifying novel molecular targets combined with efficient delivery tools to improve the current landscape of cancer therapies (7).

\section{Lipid-based NPs (LBNPs) for non-coding RNA (ncRNA) delivery in breast cancer cells}

LBNPs, and nanostructured lipid carriers (NLCs) have been recognized among a large number of non-viral vectors as alternative, effective and safe methods for gene therapy to potentially treat both genetic and non-genetic diseases, including ocular and infectious diseases, lysosomal storage disorders and cancer (8). These methods have been developed to overcome the numerous challenges, including the low stability of the formulations in the blood circulatory system, drug burst release by erosion mechanisms, immune system evasion and high toxicity, for successful gene delivery and effectiveness. LNs are able to overcome the main biological barriers for successfully transfecting cells, including the degradation of therapeutic ncRNAs by nucleases, cell internalization, intracellular trafficking and the inability to selectively target a specific cell type (8). Additionally, LNs may offer significant advantages, including an improved bioavailability by enhancing aqueous solubility, increasing the half-life for clearance, increasing the specificity for its cognate receptors and targeting drugs to specific locations in the target tissues (9).

In addition, from a safety point of view, LNs are constructed using well-tolerated components, while from the technological point of view, they may be easily produced on a large scale, and subjected to sterilization and lyophilization, thus providing the best storage stability. Liposomes are the most studied delivery systems due to their biocompatibility and biodegradability. These NPs are primarily composed of phospholipids, which are organized in bilayer-forming structures due to the amphipathic properties of the phospholipid molecules. In the presence of water, NPs form vesicles, thus improving the solubility and stability of the antitumor drugs once they are loaded into the vehicle (10). Furthermore, these lipid-based vehicles are capable of encapsulating either hydrophobic or hydrophilic drugs (8). In addition to phospholipids, other compounds, such as cholesterol, can be loaded to the formed vehicles; cholesterol decreases the membrane fluidity of the NP and increases its permeability to hydrophobic drugs, thus improving its stability in blood (10).

Several studies have demonstrated that NPs are useful and efficient tools for delivering ribonucleic acids into tumor cells and tissues. Although NPs have been used to deliver chemotherapeutic drugs, other molecules, such as ncRNAs, can be also transported into tumor cells. Therefore, NPs encompassing ncRNAs are considered as a promising alternative approach, as ncRNAs mediate the specific and potent silencing of oncogenes, and more specifically, of the tyrosine kinase receptors expressed on the tumor cell surface, as they are well-known and important drivers of carcinogenesis (11). During this process, the degradation of oncogenic mRNAs by ncRNAs is mediated by a RNA interference (RNAi) mechanism, which serves an important role in the modulation of gene expression (12). Among the aforementioned delivery systems, NLCs have been widely used for transporting chemotherapy drugs and other molecules, especially in the form of liposomal vehicles. The most frequently used nanosystems for delivering ncRNAs include the cationic, neutral and ionizable liposomes, and exosomal and other synthetic nanocarriers (Fig. 1 and Table I) (13-19). These liposomes exhibit several advantages when acting as carriers for the in vitro delivery of RNAi agents, as they can be easily prepared from biocompatible lipids or phospholipid components, and be modified to serve specific purposes, such as the encapsulation of the hydrophilic drug molecules.

The innate immune system constitutes the first line of defense and consists of a diverse set of cell types, including monocytes, macrophages and dendritic cells. These cells express different pattern recognition receptors on their cell surface, such as the membrane-bound Toll-like receptors, cytoplasmic nucleotide-binding oligomerization domain-like receptors and scavenger receptors. The adaptive immune system provides the second line of defense and includes several important Thelper (Th) cell subsets, including Th1, Th2, Th17, $\mathrm{T}$ regulatory and Th22 cells. In a mouse model of vaccination using antigen-coupled nanobeads, it was reported that the NP size determined the Th1 response (IFN $\gamma$ production) and Th2 response (IL-4 production); the 40 and $49 \mathrm{~nm}$ beads significantly improved Th1 activation, whereas the larger sized beads $(>100 \mathrm{~nm}$ ) stimulated the Th2 response to a great extent (20). These blood cells and erythrocytes interact with various types of NPs depending on their composition, size, geometry and surface charge, and importantly, these interactions may affect the delivery of NPs as they can forms aggregates, mainly with the abundant erythrocytes, and be toxic for cells of the immune system. The geometry of the NPs also defines the efficiency of the cellular absorption and the ability to form aggregates; for example, the rod-shaped NPs are internalized by leukocytes with high efficacy, followed by sphere- and cylinder-shaped NPs, whereas cube-shaped NPs are not as easily internalized (21). Regarding the charge, cationic NPs will interact positively with the surface of the negatively charged cell membrane; however, cationic liposomes can enhance immunotoxicity by stimulating neutrophils and inducing oxidative stress. In addition, anionic NPs have been shown to have unfavorable interactions with the cell membrane due to the repulsive forces between the two negatively charged surfaces and therefore, show poor cellular internalization (22). Conversely, it has been proposed that leukocyte-NP interactions are advantageous, since these complexes can easily cross physiological barriers and travel to tumor niches (23).

Cationic liposomes are especially convenient and the electrostatic interactions between the positively charged lipids and 
Table I. Summary of examples of delivery systems for therapeutic microRNAs and siRNAs in breast cancer.

\begin{tabular}{|c|c|c|c|c|}
\hline First author, year & Therapeutic agent & Example of delivery system & Cancer type & (Refs.) \\
\hline Chen et al, 2017 & siRNA & H40-P(Asp-AED-ICA)-polyethylene glycol & Breast cancer & (13) \\
\hline Deng et al, 2014 & miRNA-34a mimics & Hyaluronic acid-chitosan & Breast cancer & (14) \\
\hline Hogrefe et al, 2006 & siRNA & Immunoliposome & $\begin{array}{l}\text { Breast cancer } \\
\text { xenograft }\end{array}$ & $(15)$ \\
\hline Shu et al, 2015 & miRNA-21 inhibitor & $\begin{array}{l}\text { RNA-NPs decorated with EGFR-targeting } \\
\text { aptamer }\end{array}$ & $\begin{array}{l}\text { Triple-negative } \\
\text { breast cancer }\end{array}$ & $(16)$ \\
\hline Urban-Klein et al, 2005 & siRNA & $\begin{array}{l}\mathrm{pH} / \text { redox dual-sensitive cationic } \\
\text { unimolecular NP }\end{array}$ & $\begin{array}{l}\text { Triple-negative } \\
\text { breast cancer }\end{array}$ & $(17)$ \\
\hline Yhee et al, 2015 & siRNA & Glycol chitosan & $\begin{array}{l}\text { Drug-resistant } \\
\text { breast cancer }\end{array}$ & $(18)$ \\
\hline Guo et al, 2014 & siRNA & $\begin{array}{l}\text { 1,2-Dioleoyl-3-dimethylammonium-propane, } \\
\text { 1,2-dioleoyl-sn-glycero-3-phosphocholine, } \\
\text { 1,2-dioleoyl-sn-glycero- } \\
\text { 3-phosphoethanolamineN-dodecanoyl }\end{array}$ & $\begin{array}{l}\text { Triple-negative } \\
\text { breast cancer }\end{array}$ & (19) \\
\hline
\end{tabular}

siRNA, small interfering RNA; miRNA, microRNA; PEI, polyethylenimine; NP, nanoparticle.

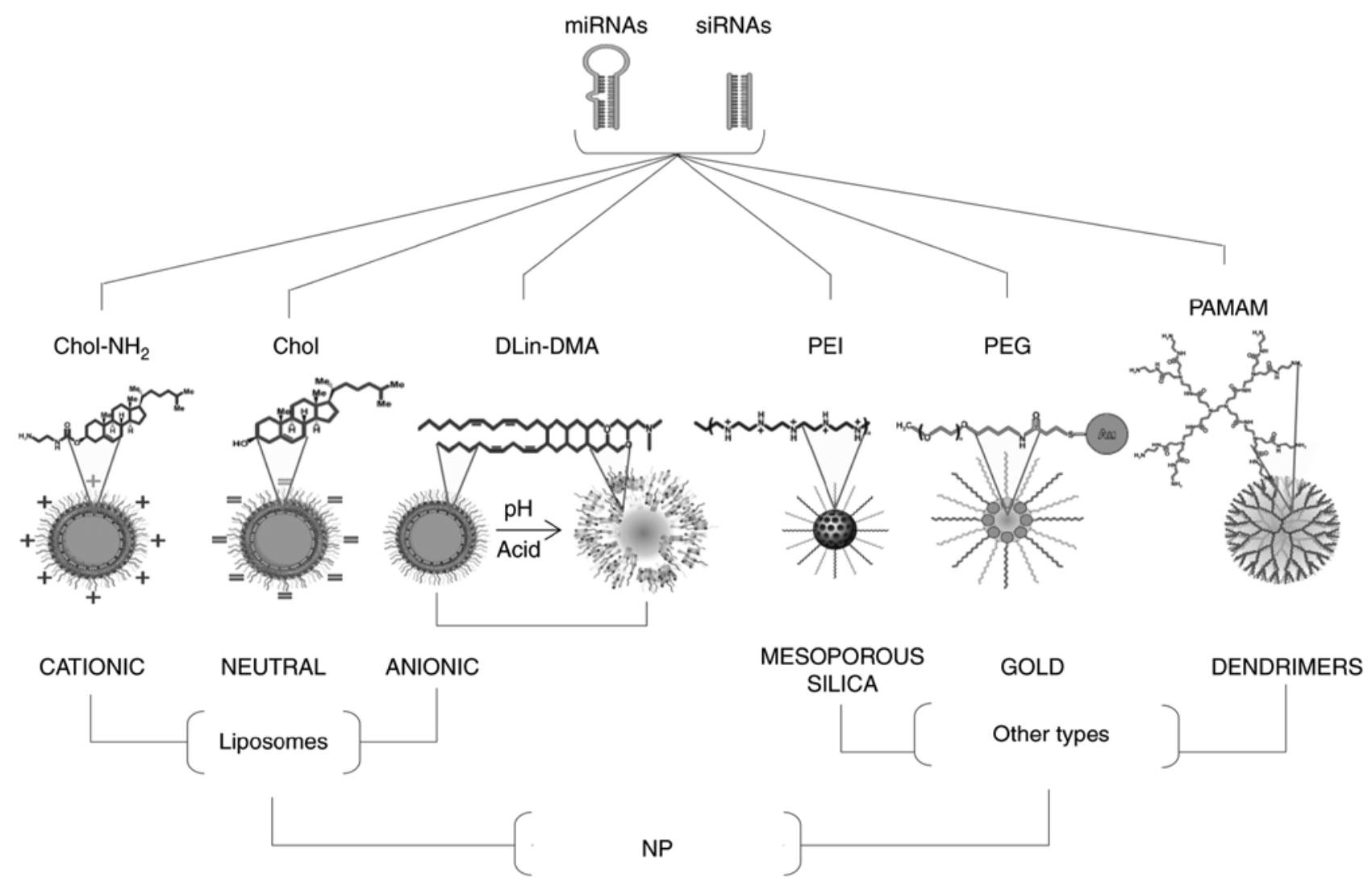

Figure 1. Graphical representation of common types of NPs composed of liposomes and other types (mesoporous silica, gold and dendrimers) for the delivery of nanomiRNAs (siRNAs or miRNAs) into tumor cells. The three kinds of liposomes known as cationic, neutral, and anionic $\mathrm{pH}$-sensitive, synthesized with Chol- $\mathrm{NH}_{2}$, Chol and DLin-DMA, respectively, are indicated on the left-hand side. On the right side are mesoporous silica, gold and dendrimers-based NPs covalently or not covalently linked to PEI, PEG and PAMAM, respectively. siRNA, small interfering RNA; miRNA, microRNA; Chol, cholesterol; DLin-DMA, N,N-Dimetilacetamida; PAMAM, poly-amido amine; PEG, polyethylene glycol; PEI, polyethylenimine; NP, nanoparticles.

the negatively charged RNA molecules notably improve the efficiency of RNA encapsulation. The encapsulated ncRNAs within cationic liposomes exhibit a preferential uptake from the target cells through endocytosis. To further ensure that the 
desired cells are targeted, ligands binding to cell surface receptors of the target tissue are incorporated into the cationic coat of the liposome (24). The engineering of carriers has gained considerable attention in recent years due to the development of effective delivery systems that transport small interfering RNAs (siRNAs) and microRNAs (miRNAs/miRs) into the tumor tissues and the cytoplasm of tumor cells. Furthermore, an ideal nanocarrier system should be able to protect the therapeutic RNAi agents from the circulatory environment, provide high resistance to nucleases, overcome immune responses and ensure efficient delivery to tumor cells (25).

An interesting study by Hayward et al (26) described the delivery of miR-125a-5p in HER2-positive metastatic breast cancer cells using a lipid-based system; the study demonstrated that LNs coated with hyaluronic acid used for miR-125a-5p delivery significantly downregulated the expression levels of HER2 and suppressed cell proliferation and migration via the PI3K/AKT and MAPK signaling pathways. Thus, the findings of this study suggested a therapeutic potential of miRNAs coupled to LNs for HER2-positive breast cancer treatment. Another study, performed in cancer stem-like cell subpopulations derived from MCF-7 breast cancer cells, demonstrated that the delivery of miR-200c by LBNPs downregulated the expression levels of class III $\beta$-tubulin protein, which is considered as a candidate biomarker for predicting resistance to chemotherapy (27). In addition, LBNPs exhibited lower cytotoxicity compared with lipofectamine in a previous study. These data illustrated the promising efficiency of LBNPs in delivering miRNAs, thus suggesting that LBNPs may be considered as a powerful therapeutic tool for the treatment of breast cancer.

Furthermore, Wang et al (28) evaluated the efficiency of LNs in delivering specific siRNAs targeting CDK4; the results revealed that the $\mathrm{LN}$ triggered exceptional gene silencing, to a greater extent than that of commercial lipofectamine. CDK4 silencing in HeLa cervical cancer cells and MDA-MB-468 breast cancer cells also promoted $\mathrm{G}_{1}$ phase cell cycle arrest. Therefore, as CDK4 is involved in the cell cycle and proliferation, the development of selective blocking agents against CDK4, such as siRNAs, is considered a very attractive approach for cancer treatment. In addition, Tang et al (29) explored the efficiency of an interesting delivery system, in which LNs were coated with calcium phosphate, in transferring a mixture of highly potent siRNAs targeting ubiquitously expressed genes essential for cell survival. The results reported enhanced cellular uptake and the inhibition of MDA-MB-468 breast cancer cell growth. Furthermore, the conjugation of either EGFR-specific single chain fragment antibody or folic acid improved the delivery ability of the siRNAs into the cancer cells.

The simultaneous delivery of chemotherapeutic drugs and therapeutic ncRNAs has provided remarkable advantages; for example, Yu et al (30) developed a co-delivery system consisting of paclitaxel and a specific siRNA targeting myeloid cell leukemia-1 (MCL1) coupled to LBNPs; the results of this study demonstrated that MCL1 mRNA expression levels were significantly downregulated in MDA-MB-231 cancer cells transfected using the aforementioned delivery system. Additionally, in a xenograft mouse model, the intratumoral injection of LNs encapsulating paclitaxel and siRNA-MCL1 significantly attenuated tumor growth. These results indicated that nanomiRNAs may efficiently transport antitumor and therapeutic drugs.

The high specificity and performance of the cellular RNAi mechanism may explain the above effects. Since the discovery of RNAi in mammalian cells, there has been increasing interest in harnessing this mechanism for treating several types of disease including ophthalmologic disease, cancer, metabolic diseases such as hypercholesteremia, and viral infections. Briefly, RNAi is an endogenous pathway associated with the post-transcriptional silencing of gene expression, triggered by small double-stranded RNAs (dsRNAs) functioning in a sequence-specific manner via directly binding and degrading target mRNAs, thus leading to the potent silencing of gene expression (31). miRNAs are small ncRNAs of 21-25 nucleotides in length, encoded by genes dispersed throughout the genome. These tiny RNAs act at the post-transcriptional level as negative regulators of gene expression through binding to the 3'-untranslated region of multiple mRNAs, thus leading in their degradation and transcriptional repression via the activation of the cellular RNAi mechanism (32). Interestingly, miRNAs may function as oncogenes or tumor suppressors, regulating the expression of a number of genes involved in pivotal signaling pathways and cellular processes that promote tumor development and progression, including cell proliferation, the cell cycle, angiogenesis, the cancer stem cell-like phenotype, epithelial-mesenchymal transition, and invasion and metastasis (33-39). The expression levels of miRNAs are often dysregulated in different types of tumor and their ability to directly target multiple genes greatly affects the cancer cell phenotype, thus resulting in tumorigenesis $(40,41)$. These cellular alterations on the expression profile of miRNAs determine the clinicopathological features of patients, resulting in a decreased overall survival and disease-free survival rates, and an increased risk of disease relapse, drug resistance $(42,43)$, disease recurrence (44) and poor prognosis (45). Therefore, miRNAs may serve as bona fide diagnostic and predictive biomarkers for several types of disease, including obesity, neurological disorders, cardiopathies and cancer (46). On the other hand, siRNAs are a class of small dsRNAs, which are exogenously administered into cells, as they are not encoded in the genome, to mediate gene silencing. siRNAs inhibit gene expression and protein synthesis via complementary binding to their target mRNAs, where they trigger target gene silencing through the RNAi mechanism, like miRNAs (47). A conventional siRNA consists of 19-21 nucleotides in length with a 3 ' double-nucleotide overhang, usually TT and UU; these nucleotides are important for sequence recognition by the RNAi machinery. As the length of the dsRNA increases, its potency is significantly enhanced. For instance, an in vitro study demonstrated that siRNAs of 27 nucleotides in length were up to 100 times more potent compared with the conventional 21-nucleotide siRNAs (48). In recent years, novel strategies for the targeted delivery of miRNAs and siRNAs into tumor cells have been developed; however, the primary barrier to the clinical application of these RNAi-mediated therapies remains the lack of an effective delivery system providing sufficient protection for the ribonucleic acids against nucleases. Among them, nanomiRNAs offer great potential owing to their biocompatibility and low toxicity compared 
with inorganic NPs and viral delivery systems (49). However, to the best of our knowledge, the development of clinical trials using ncRNA delivery systems through NPs has not yet been explored in patients with breast cancer. Two registered clinical trials using different approaches to deliver therapeutic miRNAs in different cohorts of patients with cancer have been reported. The first report is a phase I study of TargomiRs as 2nd or 3rd line treatment for patients with recurrent malignant pleural mesothelioma (MPM) and non-small cell lung cancer (clinical trial no. NCT02369198). TargomiRs are targeted minicells (bacteria-derived NPs) containing the tumor suppressor miR-16 mimic, which is a small RNA involved in diverse types of cancer. The drug delivery system denominated EnGeneIC Delivery consists of nonliving bacterial minicells forming NPs of 100-400 nm size and the TargomiRs system is targeted to tumors using an anti-EGFR antibody. The data illustrated that the TargomiRs formulation displayed acceptable safety and was well tolerated by the 26 patients with refractory MPM enrolled in the study; however, it only promoted moderate antitumor activity (50). The second report is a multicenter phase I study using a miR-34a mimic-encapsulated liposomal formulation (MRX34), which was injected into 85 participants with diverse types of solid tumor, including hepatocellular carcinoma, melanoma, renal carcinoma and lung cancer, among others (clinical trial no. NCT01829971). The pharmacodynamic data indicated the efficient delivery of miR-34a into the tumors, and a dose-dependent and longer-lasting repression of its cognate target genes $(\mathrm{Bcl}-2$, DnaJ homolog subfamily B member $1, \beta$-catenin, forkhead box protein $\mathrm{P} 1$ and histone deacetylase 1) in white blood cells (51). In addition, a manageable toxicity profile and modest clinical activity was observed following MRX34 treatment. Unfortunately, the clinical trial was closed due to unexpected adverse events related to the immune system that were not anticipated in pre-clinical studies (52). At present, these novel nanotechnologies are gaining increasing attention as promising anticancer tools due to their high specificity, efficiency and low toxicity in normal cells $(53,54)$. In the next section, the current applications of diverse nanomiRNAs systems in breast cancer are summarized.

\section{Cationic liposomes}

The lipids constituting the cationic liposomes neutralize the negative charge of the DNA, thus forming a compact structure, which differs from the typical vesicular structure of liposomes. These complexes, resulting from the interaction between DNA and the cationic lipids, provide protection to the genetic material and promote cell internalization. For gene therapy applications, the surfactants are often positively charged to obtain cationic LBNPs electrostatically bound to nucleic acids (55). In addition, LBNPs usually exhibit a $\zeta$ potential, an indirect measurement of the surface charge $(>30 \mathrm{mV})$ which decreases following the addition of increasing concentrations of nucleic acids (10). Previous studies have taken advantage of the features of cationic liposomes to mediate the silencing of cancer-related genes in different types of cancer; for instance, Meraz et al (56) demonstrated in a murine model of breast cancer that the intratumoral injection of cationic liposomes loaded with monophosphoryl lipid A and IL-12 promoted cell death, inhibited cell proliferation and increased the serum levels of IL-1 $\beta$ and TNF- $\alpha$. Thus, this study illustrated the efficacy of liposomal nanocarriers to attenuate cell viability, tumor growth and drug toxicity, and enhance immunity.

Other researchers have implemented innovative systems for the simultaneous delivery of siRNAs and chemotherapeutic agents, including cationic liposomes $(57,58)$. Such a system was applied in breast cancer, where cationic liposomes were used to deliver paclitaxel together with a specific siRNA targeting polo-like kinase 1 (PLK1). PLK1 is an aggressive oncogene frequently found overexpressed in breast cancer, thus promoting the accelerated proliferation of tumor cells. The simultaneous delivery of paclitaxel and PLK1 siRNA synergistically increased the number of apoptotic MCF-7 breast cancer cells and reduced angiogenesis. This delivery method exhibited significant advantages over monotherapies with paclitaxel and siRNAs (57).

On the other hand, the conjugation of chemotherapeutic agents, such as doxorubicin, with liposomes is a commonly used method in cancer treatment (58). Wang et al (58) modified the doxorubicin-liposome system with a cationic polymer to improve the cellular absorption and antitumor activity of NPs; the NPs were efficiently absorbed, resulting in cell death following $5 \mathrm{~h}$ incubation compared with doxorubicin monotherapy and doxorubicin-liposomes complexes. In addition, the efficacy of the above delivery system was determined in $\mathrm{H} 22$ mice following four injections of doxorubicin-liposomes conjugated to a cationic polymer; the results revealed that the delivery system conjugated to the cationic polymer reduced tumor growth by up to $60 \%$ in vivo. Thus, the aforementioned studies supported the great potential of cationic liposomes to improve the delivery of anticancer drugs.

\section{Neutral liposomes}

Neutral liposomes are primarily constructed by neutral lipids, including phosphatidylcholine, phosphatidylethanolamine, cholesterol and 1,2-dioleoyl-sn-glycero-3-phosphoethanolamine (DOPE) $(59,60)$. DOPE may be used as an adjuvant phospholipid in cationic liposomes and it was discovered to enhance the transfection efficiency of nanocarriers encapsulating nucleic acids (59). In addition, neutral liposomes have exhibited good biocompatibility and excellent pharmacokinetic characteristics; however, they are unable to interact with DNA to efficiently adsorb and encapsulate it into the liposomes $(59,60)$. Yan et al (61) constructed a novel and functional type of neutral liposomes; these neutral liposomes, known as 1,2-distearoyl-sn-glycero-3-phosphoeth anolamine-N-[(polyethylene glycol)-2000], efficiently silenced the expression levels of Slug and inhibited the TGF- $\beta 1 /$ Smad signaling pathway in triple-negative breast cancer cells. In addition, these liposomes demonstrated a high antitumor efficacy in vivo when combined with vinca alkaloid (vinorelbine) chemotherapy in triple-negative breast cancer. Another study revealed that pegylated liposomal doxorubicin significantly extended the disease-free survival period of patients with pathological stage I-III breast cancer, which suggested that it could be applied at different pathological stages of breast cancer (62).

Emerging evidence has suggested that curcumin may overcome the drug resistance to conventional chemotherapies; 
however, its poor bioavailability and decreased absorption have limited its clinical use $(63,64)$. Furthermore, adriamycin, a widely known cytotoxic agent, is often used in cancer chemotherapy (65); however, breast tumors have exhibited high resistance to adriamycin, leading to a poor prognosis. Zhou et al (66) developed NPs encapsulating the dietary compound curcumin to improve its bioavailability. Furthermore, miRNA profiling was performed in adriamycin (Adr)-resistant MCF-7 breast cancer cells (MCF-7/Adr) and the results revealed that neutral liposomes containing curcumin increased the chemosensitivity of MCF-7/Adr cells, which was mediated by the altered expression pattern of miRNAs associated with drug resistance. In fact, 67 differentially expressed miRNAs were identified among the parental MCF-7, MCF-7/Adr and curcumin-treated MCF-7/Adr cells; among them, the downregulation of miR-29b-1-5p was associated with decreased $\mathrm{IC}_{50}$ values of curcumin, while its upregulation attenuated the effects of liposomal curcumin in Adr-resistance in MCF-7/Adr cells.

Another previous study described the development of a functional neutral liposome for miR-203 delivery; these miR-203-containing liposomes efficiently silenced Slug expression levels and inhibited the TGF- $\beta 1 /$ Smad signaling pathway in triple-negative breast cancer cells. Additionally, when liposomes were combined with vinorelbine chemotherapy, they exhibited significant antitumor activity in triple-negative breast cancer cells. Therefore, these findings suggested that the development of functional miRNAs and neutral liposomes should be further investigated in clinical trials (61).

Furthermore, it was revealed that the growth inhibitory effects of siRNAs targeting VEGF loaded into liposomes incorporating modified $\mathrm{G} 2$ polyamidoamine-cholesterol dendrimer were comparable with those noted to commercially available transfection systems (Metafectene) in SKBR3 breast cancer cells (67). In addition, Chen et al (68) applied a novel polycation liposome encapsulating a calcium phosphate (PLCP) NP to facilitate endosomal escape via combining the protonation of polyethylenimine and membrane destabilization of DOPE, which resulted in an increased transfection efficiency. In addition, this siRNA delivery system was also applied using three siRNA sequences targeting VEGF to inhibit tumor angiogenesis. Compared with the commercial transfection reagents, the silencing activity of the PLCP/VEGF siRNA complex was increased two times in MCF-7 cells. In vivo results in an MCF-7 xenograft mouse model revealed that the PLCP/VEGF siRNA complex significantly inhibited angiogenesis and tumor growth. Additionally, a synergetic tumor inhibition effect was observed in cells co-treated with doxorubicin in a mouse model. Thus, the aforementioned data suggested that the delivery of siRNAs targeting VEGF in combination with PLCP inhibited angiogenesis, indicating that this delivery system may serve as a promising approach for breast cancer treatment.

\section{Ionizable liposomes}

Ionizable liposomes are promising nanocarriers used to deliver siRNAs due to their physical and functional properties. Unlike the cationic and neutral liposomes that carry a single type of charge, ionizable liposomes are protonated and deprotonated according to the acidity of the environment (59). Early cationic lipids, such as N-[1-dioleyloxy)propyl]-N,N,N-trimethylammonium and dioctadecyldimethylammonium chloride, contain a positive quaternary amine, whereas ionizable cationic lipids, such as 1,2-dioleoyl-3-dimethylammonium propane and 1,2-dioleyloxy-N,N-dimethyl-3-aminopropane, have positive and neutral charged tertiary amines, respectively, at acidic and physiological $\mathrm{pH}$ conditions (69). Furthermore, it has been reported that the incorporation of helper and neutral lipids, such as cholesterol and saturated phosphatidylcholines, increase both the system stability and transfection efficiency (70). The effects of the ionizable liposomes in cancer therapy have been poorly investigated; however, experimental evidence has suggested that their application provides significant advantages compared with other types of therapeutic methods (71). For example, an in vivo study in a glioma murine model discovered that the delivery of conjugated siRNAs to ionizable liposomes exhibited an improved efficiency and resulted in enhanced cellular uptake under hypoxia and low-pH conditions. Specifically, in this study, Liu et al (72) synthesized malate dehydrogenase lipids with nitroimidazole groups, which provided hypoxia sensitivity and specificity as hydrophobic tails, and tertiary amines as hydrophilic head groups. These precursors were self-assembled into O'1,O1-(3-(dimethylamino) propane-1,2-diyl) 16-bis(2-(2-methyl-5-nitro- $1 H$-imidazol1-yl)ethyl) di(hexadecanedioate) liposomes (MLP) to encapsulate PLK1 siRNA; the results demonstrated the enhanced delivery of MLP/PLK1 siRNAs into glioma cancer cells due to the increased positive charges induced by hypoxia and low $\mathrm{pH}$. Therefore, MLP/PLK1 siRNA was shown to effectively inhibit glioma cell growth both in vitro and in vivo.

In addition, it has been reported that the implementation of ionizable liposomes increases encapsulation (-2,200 molecules per $100 \mathrm{~nm}$ liposome), whereas the therapeutic effects of encapsulated siRNAs and their useful shelf life are greater compared with another liposomes $(73,74)$. Active loading remains the most commonly used method for loading ionizable drugs in liposomes; however, the low aqueous solubility of numerous anticancer drugs may slow liposomal uptake during active loading (74). To overcome this limitation, drug supersaturation is maintained during active loading (73). Modi et al (73) developed a method for maintaining supersaturation of a poorly soluble camptothecin drug analogue, namely 7-t-butyldimethylsilyl-10-hydroxycamptothecin, using low concentrations of sulfobutylether- $\beta$-cyclodextrin to inhibit crystallization, thus overcoming the limitations associated with the liposomal delivery of poorly soluble ionizable drugs.

It has been reported in a breast cancer model that the application of ionizable liposomes with vincristine at a drug-lipid ratio of 1:1 provided an average life of $15.7 \mathrm{~h}$ when combined with conventional cyclophosphamide, doxorubicin, vincristine and prednisone chemotherapy. Therapeutically optimized rates of drug release were suggested to be achieved by varying the drug-to-lipid ratios in liposomal vincristine formulations (75). Another study demonstrated the implementation of ionizable liposomes conjugated to lipocalin 2 (Lcn2) siRNA to inhibit the C-X-C chemokine receptor type 4 and Lcn2 pathways in metastatic MDA-MB-436 and MDA-MB-231 breast cancer cell lines, 
thus indicating that the treatment with liposomes may inhibit breast cancer progression (19).

At present, studies with liposome-miRNA complexes at the nanometer and micrometer scale are being conducted for their pharmaceutical application in breast cancer. Defining the critical quality attributes of nanosystems using high level quality control for reproducibility is pivotal for their potential application in cancer research (76). Lujan et al (76) studied diverse aspects of the formation of nanoliposomal systems for the proper storage and delivery of miRNAs in triple-negative breast cancer cells; the study revealed that the nanometer-sized liposomes $(<350 \mathrm{~nm})$ could be obtained at low concentrations, preserved stably for up to 6 months in lyophilized form and maintained encapsulation after extended time periods in storage. Furthermore, the nano-formulation efficiency was determined by delivering miR-203, a tumor suppressor, into MDA-MB-231 and Hs578t triple-negative breast cancer cells. The results illustrated that both micro- and nanoliposomes effectively delivered miR-203 into breast cells via an endocytic pathway. Another previous study in a triple-negative breast cancer mouse model revealed that the encapsulation and administration of miR-203 in liposomes of a size of $120 \mathrm{~nm}$ inhibited tumor growth compared with functional vinorelbin liposomes. Remarkably, the treatment with both types of liposomes resulted in complete tumor growth inhibition (61).

An in vitro study by de Antonellis et al (77) focused on the restoration of the Notch signaling pathway by employing a stable 2'-O-methylated-encapsulated miR-199b-5p in ionizable lipids. miR-199b-5p was identified to regulate basic-helix-loop-helix transcription factor 1 (Hes-1), a downstream effector of the canonical Notch pathway, by affecting medulloblastoma cancer stem cells (CSCs) through decreasing the number of $\mathrm{CD} 133^{+} / \mathrm{CD} 15^{+}$cell subpopulations. The efficacy of the miR-199b-5p delivery using stable nucleic acid lipid particles was confirmed by the significant attenuation of Hes-1 and CSC marker expression levels in tumorigenic cell lines from colon (HT-29, CaCo-2 and SW480), breast (MDA-MB231T and MCF-7) and prostate (PC-3) cancer, glioblastoma (U-87) and medulloblastoma (Daoy, ONS-76 and UW-228). In addition, the formulation inhibited cell proliferation, thus indicating that this system could be considered as a powerful tool for gene therapy. Furthermore, it was revealed that hypoxia induced in solid tumors efficiently favored the ionizable liposomes to transfer effector particles (72). Therefore, these studies on liposomes may represent a potential research approach for breast cancer $(70,72)$.

\section{Exosomes nanocarriers}

Exosomes are extracellular small vesicles present in the circulation, with diameters ranging from 30 to $150 \mathrm{~nm}$. Exosomes serve an important role in cell-to-cell communication and in the regulation of several biological processes in normal cells (78). However, in cancer cells, this cellular communication process is exacerbated, as cancer cells secrete 10-fold more exosomes, the so-called tumor-derived exosomes (TDEs). TDEs carry growth factors, DNA, chemokines and ncRNAs to promote cell communication and contribute to the tumor microenvironment, metastasis, tumorigenesis and cancer metabolism $(79,80)$. Xin et al $(81)$ demonstrated that miR-455-5p was specifically overexpressed in tumor exosomes; likewise, the exosomal and cellular expression of miR-1255a was also upregulated. It was reported that $\mathrm{miR}-455-5 \mathrm{p}$ targeted $\mathrm{CDK}$ inhibitor $1 \mathrm{~B}$ to regulate the cell cycle, while miR-1255a regulated SMAD4 to inhibit the TGF- $\beta$ signaling pathway. Upregulated expression levels of miR-455-5p and miR-1255a were associated with a poor overall survival, while the upregulation of their target genes has been associated with an excellent overall, recurrence-free and distant metastasis-free survival. In conclusion, this study preliminarily indicated that exosomal miR-455-5p and miR-1255a may be considered as novel therapeutic targets for breast cancer.

Another previous study suggested that breast cancer-derived exosomes may contribute to tumor growth, angiogenesis, invasion and metastasis $(82,83)$. Therefore, Lin et al $(83)$ suggested that exosomes derived from human mesenchymal stem cells may promote MCF-7 breast cancer cell migration and proliferation via altering the gene expression profiles. In addition, exosomes have been indicated to also affect the microenvironment to promote tumorigenesis (82-84). A previous study also revealed differences in the miRNA expression profile between patients with triple-negative breast cancer and healthy controls; for example 8 exosomal miRNAs (miR-21, miR-141, miR-200a, miR-200b, miR-200c, miR-203, miR-205 and miR-214) from malignant tumors were significantly differentially expressed from those observed in benign tumors, and were not detected in normal controls (85). In addition, the levels of exosomal let-7f and/or miR-30e-3p in patients with non-small cell lung cancer could distinguish patients with resectable tumors from those with non-resectable tumors (86). The expression levels of 12 exosomal miRNAs (miR-17-3p, miR-21, miR-106a, miR-146, miR155, miR-191, miR-192, miR-203, miR-205, miR-210, miR-212 and miR-214) were significantly different between patients and controls (87). The differences in miRNAs expression exhibited promising predictive values; thus they may serve as biomarkers to distinguish patients with recurrent breast cancer from patients with non-recurrent breast cancer (88).

\section{Stealth liposomes}

Stealth liposomes, also called PEG-coated liposomes, exhibit prolonged blood circulation time and improved distribution in tissues. This type of liposome is characterized by the incorporation of a PEG derivative, which promotes the steric stabilization of NPs to overcome their opsonization and prolong their time in the blood circulation (89). In addition, the presence of PEG on the surface of liposomes was discovered to increase the hydrophilicity, thus reducing their interaction ability with plasma proteins $(90,91)$. PEG anchoring can be obtained by the polymer adsorption into vesicles and aggregation of the PEG-lipid during development (92). These lipids exhibit several advantages, including increased solubility, null toxicity and low antigenicity. Furthermore, for practical purposes, it is easier to incorporate this type of polymer with lipids. Another interesting advantage is that PEG lipids have been reported to potentiate therapeutic proteins without altering their mechanism of action (93). At present, a pegylated liposome loaded with doxorubicin, DOXIL ${ }^{\circledR}$ has been approved by the Food and Drug 
Administration since 1995. These nanodrugs exhibit prolonged circulation time, as they can overcome their recognition by the reticuloendothelial system due to the presence of PEG on the surface of the lipids. Furthermore, loading of these liposomes with doxorubicin, directed by an ammonium sulfate gradient, improved the delivery of the drug into tumor cells (94). A study by Vakhshiteh et al (95) demonstrated that the encapsulation of miR-34a into a type of PEG-anchored liposomes promoted its accumulation in MDA-MB-231 breast carcinoma cells. Additionally, this formulation exhibited a significant inhibitory effect on tumor cell growth, migration and invasion, whereas it attenuated the percentage of $\mathrm{CD} 44^{+} / \mathrm{CD} 24^{-}$cells, indicating its effectiveness in cancer hallmarks.

\section{Triggered-release liposomes}

Liposomes are NPs widely used for the delivery of drugs, DNA fragments and siRNAs, which allow the release of these molecules in time and space (96). The activated liposomes represent a promising system that may increase the release rate of their contents (97). The ability to deliver antitumor molecules in situ remains a challenge for the development of liposomes, thus several strategies have been applied considering the physical and chemical characteristics of the tumor microenvironment $(98,99)$. For example, Elegbede et al (100) developed an interesting formulation with a collagen-like triple-helix conformation encompassing a matrix metalloproteinase (MMP) substrate to induce drug release in a MMP activity-dependent manner. Another study revealed that thermosensitive liposomes, used for localized delivery, triggered the release of chemotherapeutic drugs. For example, the delivery of pegylated liposome-based drugs, like DOXIL, was more efficient in tumors exposed to slightly higher microenvironment temperatures (101). In addition, an in vitro study performed in triple-negative breast cancer cells showed that a benzoporphyrin-derived conjugate exhibited a lifespan of one month at $37^{\circ} \mathrm{C}$, suggesting that it could be successfully employed for in vivo interventions (102).

\section{Porphysomes}

Phosphorene, also referred to as single- or few-layer black phosphorus (FLBP), is a new member of the 2D material family (103). FLBPs have gained increasing attention in recent years and have been used in optoelectronics, strength storage and biomedicine due to their efficiency and biocompatibility (104). In 2017, Yang et al (103) constructed FLBP nanosheets loaded with gold $(\mathrm{Au})$-NPs and black phosphorus (BP)-NPs as effective surface-enhanced Raman scattering (SERS) substrates; the features of the breast tumors before and after photothermal therapy were distinguished utilizing the SERS analysis and $>85 \%$ of $4 \mathrm{~T} 1$ cells were alive following incubation with BP-Au NSs, thus indicating that the hybrid NP BP-Au exhibited good biocompatibility and low cytotoxicity. Another advancement originated from the study by Sun et al (105), who focused on the application of photodynamic treatment (PDT) combined with quality treatment for triple-negative breast cancer. The objective was accomplished by utilizing cationic porphyrin lipid microbubbles loaded with hypoxia-inducible factor- $1 \alpha$ (HIF-1 $\alpha)$ siRNA, which was monitored using ultrasound imaging. HIF-1 $\alpha$ siRNA downregulated HIF-1 $\alpha$ expression levels, which was mediated by the common hypoxic tumor conditions or the reactive oxygen species (ROS) produced by PDT, improved the PDT efficacy and partially inhibited tumor progression.

\section{Lipid-coated calcium phosphate (LCP) NPs}

In 2018, Tang et al (106) designed and developed biocompatible multifunctional LCP NPs as an effective delivery system to inhibit the development of tumors using triple-negative MDA-MB-468 cells. Briefly, LCP NPs were conjugated with a bispecific immunizer (BsAb) through a non-covalent bond with methoxy PEG on the surface of the molecule; this BsAb targeted the EGFR on the surface of MDA-MB-468 cells. These LCP-BsAb NPs, loaded with cell death (CD) siRNA and indocyanine green (ICG), were efficiently uptaken by MDA-MB-468 cells, promoting cell apoptosis and cell absorbance at $808 \mathrm{~nm}$ using a near-infrared laser. These multifunctional LCP-BsAb NPs were increasingly accumulated in the tumor tissue. Thus, the combination of CD siRNA and photothermal (ICG) treatment using LCP-BsAb NPs inhibited both small and large tumors in the in vivo mouse model $(106,107)$.

\section{Conclusions}

In conclusion, the recent advances in nanotechnology and the development of novel LBNPs highlight the potential for its utilization in cancer therapy. Treatments using LBNPs greatly improve the transport of ncRNAs into cancer cells and tumor tissues and at the same time, demonstrated significant advantages, such as reduced therapeutic doses, low cytotoxicity to normal cells and an ability to reverse the resistance to chemotherapy. Moreover, LBNPs have the ability to transport therapeutic ncRNAs to modulate the expression levels of genes involved in cell growth, proliferation, cell death, invasion and metastasis, thereby impairing the malignant behavior of tumors. Therefore, the development of efficient nanomiRNAs in breast cancer will depend on the proper synthesis of new lipid components and novel targeting ligands. LBNPs are promising tools for the therapeutic delivery; however, although significant advancements have been made in the field of ncRNA delivery, there still exists a further requirement to investigate alternative effective strategies. LBNPs also have disadvantages; for example, due to their crystalline structure, they have low drug loading efficiency and burst release. Also, there is a high probability for drug expulsion from the NPs due to the crystallization process during the storage conditions, where polymorphic changes between the LBNPs and compounds promotes the tendency for drug expulsion. Undoubtedly, nanotechnology-based treatments are still far from reaching the patients; however, it is a treatment that, together with the development and increased research into different microRNAs, may serve a fundamental role in the non-invasive treatment of multiple types of cancer.

\section{Acknowledgements}

We would like to thank Mr. Juan Manuel Delgado Cervantes at the Facultad de Medicina de la Universidad Autónoma de San Luis Potosí for the support in producing the figure. 


\section{Funding}

No funding was received.

\section{Availability of data and materials}

Not applicable.

\section{Authors' contributions}

MBSC, MZSL, EJA, SINO, ICM and CLC wrote all the sections of manuscript. CLC and MBSC conceived and designed the review. All authors read and approved the final manuscript.

\section{Ethics approval and consent to participate}

Not applicable.

\section{Patient consent for publication}

Not applicable.

\section{Competing interests}

The authors declare that they have no competing interests.

\section{References}

1. Cavallo F, Giovanni C, Nanni P, Forni G and Lollini PL: 2011: The immune hallmarks of cancer. Cancer Immunol Immunother 60 : 319-326, 2011.

2. Bray F, Ferlay J, Soerjomataram I, Siegel RL, Torre LA and Jemal A: Global cancer statistics 2018: GLOBOCAN estimates of incidence and mortality worldwide for 36 cancers in 185 countries. CA Cancer J Clin 68: 394-424, 2018.

3. Cronin KA, Lake AJ, Scott S, Sherman RL, Noone AM, Howlader N, Henley SJ, Anderson RN, Firth AU, Ma J, et al: Annual report to the nation on the status of cancer, part I: National cancer statistics. Cancer 124: 2785-2800, 2018.

4. Breast Cancer Treatment/Treatment Options for Breast Cancer.

5. Miller KD, Nogueira L, Mariotto AB, Rowland JH, Yabroff KR, Alfano CM, Jemal A, Kramer JL and Siegel RL: Cancer treatment and survivorship statistics, 2019. CA Cancer J Clin 69: 363-385, 2019.

6. Hanahan D and Weinberg RA: The hallmarks of cancer. Cell 100: 57-70, 2000

7. Hanahan D and Weinberg RA: Hallmarks of cancer: The next generation. Cell 144: 646-674, 2011.

8. Yingchoncharoen P, Kalinowski DS and Des Richardson R: Lipid-based drug delivery systems in cancer therapy: What is available and what is yet to come. Pharmacol Rev 68: 701-787, 2016.

9. Mudshinge SR, Deore AB, Patil S and Bhalgat CM: Nanoparticles: Emerging carriers for drug delivery. Saudi Pharm J 19: 129-141, 2011.

10. Del Pozo-Rodríguez A, Solinís MÁ and Rodríguez-Gascón A: Applications of lipid nanoparticles in gene therapy. Eur J Pharm Biopharm 109: 184-193, 2016.

11. Hashemi M and Kalalinia F: Application of encapsulation technology in stem cell therapy. Life Sci 143: 139-146, 2015.

12. Aagaard L and Rossi JJ: RNAi therapeutics: Principles, prospects and challenges. Adv Drug Deliv Rev 59: 75-86, 2007.

13. Chen G, Wang Y, Xie R and Gong S: Tumor-targeted $\mathrm{pH} /$ redox dual-sensitive unimolecular nanoparticles for efficient siRNA delivery. J Control Release 259: 105-114, 2017.

14. Deng X, Cao M, Zhang J, Hu K, Yin Z, Zhou Z, Xiao X, Yang Y, Sheng $\mathrm{W}, \mathrm{Wu}$ Y and Zeng Y: Hyaluronic acid-chitosan nanoparticles for co-delivery of MiR-34a and doxorubicin in therapy against triple negative breast cancer. Biomaterials 35: 4333-4344, 2014.
15. Hogrefe RI, Lebedev AV, Zon G, Pirollo KF, Rait A, Zhou Q, Yu W and Chang EH: Chemically modified short interfering hybrids (siHYBRIDS): Nanoimmunoliposome delivery in vitro and in vivo for RNAi of HER-2. Nucleosides Nucleotides Nucleic Acids 25: 889-907, 2006.

16. Shu D, Li H, Shu Y, Xiong G, Carson WE III, Haque F, Xu R and Guo P: Systemic delivery of Anti-miRNA for suppression of triple negative breast cancer utilizing RNA nanotechnology. ACS Nano 9: 9731-9740, 2015.

17. Urban-Klein B, Werth S, Abuharbeid S, Czubayko F and Aigner A: RNAi-mediated gene-targeting through systemic application of polyethylenimine (PEI)-complexed siRNA in vivo. Gene Ther 12: 461-466, 2005.

18. Yhee JY, Song S, Lee SJ, Park SG, Kim KS, Kim MG, Son S, Koo H, Kwon IC, Jeong JH, et al: Cancer-targeted MDR-1 siRNA delivery using self-cross-linked glycol chitosan nanoparticles to overcome drug resistance. J Control Release 198: 1-9, 2015.

19. Guo P, You JO, Yang J, Di J, Moses MA and Auguste DT: Inhibiting metastatic breast cancer cell migration via the synergy of targeted, pH-triggered siRNA delivery and chemokine axis blockade. Mol Pharm 11: 755-765, 2014.

20. Reichmuth AM, Oberli MA, Jaklenec A, Langer $R$ and Blankschtein D: mRNA vaccine delivery using lipid nanoparticles. Ther Deliv 7: 319-334, 2016.

21. de la Harpe K, Kondiah P, Choonara Y, Marimuthu T, du Toit L and Pillay V: The hemocompatibility of nanoparticles: A review of cell-nanoparticle interactions and hemostasis. Cells 8: 1209 , 2019.

22. Buzea C, Pacheco II and Robbie K: Nanomaterials and nanoparticles: Sources and toxicity. Biointerphases 2: MR17-MR71, 2007.

23. Huang Y, Gao X and Chen J: Leukocyte-derived biomimetic nanoparticulate drug delivery systems for cancer therapy. Acta Pharm Sin B 8: 4-13, 2018.

24. Huynh A, Madu CO and Lu Y: siRNA: A promising new tool for future breast cancer therapy. Oncomedicine 3: 74-81, 2018.

25. Singh A, Trivedi P and Jain NK: Advances in siRNA delivery in cancer therapy. Artif Cells Nanomed Biotechnol 46: 274-283, 2018.

26. Hayward SL, Francis DM, Kholmatov $P$ and Kidambi S: Targeted delivery of MicroRNA125a-5p by engineered lipid nanoparticles for the treatment of HER2 positive metastatic breast cancer. J Biomed Nanotechnol 12: 554-568, 2016.

27. Liu J, Meng T, Ming Y, Wen L, Cheng B, Liu N, Huang X, Hong Y, Yuan $\mathrm{H}$ and $\mathrm{Hu} \mathrm{F}$ : MicroRNA-200c delivered by solid lipid nanoparticles enhances the effect of paclitaxel on breast cancer stem cell. Int J Nanomedicine 11: 6713-6725, 2016.

28. Wang X, Yu B, Wu Y, Lee RJ and Lee LJ: Efficient down-regulation of CDK4 by novel lipid nanoparticle-mediated siRNA delivery. Anticancer Res 31: 1619-1626, 2011.

29. Tang J, Howard CB, Mahler SM, Thurecht KJ, Huang L and $\mathrm{Xu} \mathrm{ZP}$ : Enhanced delivery of siRNA to triple negative breast cancer cells in vitro and in vivo through functionalizing lipid-coated calcium phosphate nanoparticles with dual target ligands. Nanoscale 10: 4258-4266, 2018

30. Yu YH, Kim E, Park DE, Shim G, Lee S, Kim YB, Kim CW and Oh YK: Cationic solid lipid nanoparticles for co-delivery of paclitaxel and siRNA. Eur J Pharm Biopharm 80: 268-273, 2012.

31. Zheng ZM, Tang S and Tao M: Development of resistance to RNAi in mammalian cells. Ann NY Acad Sci 1058: 105-118, 2005.

32. Guo H, Ingolia NT, Weissman JS and Bartel DP: Mammalian microRNAs predominantly act to decrease target mRNA levels. Nature 466: 835-840, 2010.

33. Yang W, Lee DY and Ben-David Y: The roles of microRNAs in tumorigenesis and angiogenesis. Int $\mathrm{J}$ Physiol Pathophysiol Pharmacol 3: 140-155, 2011.

34. Kwon T, Chandimali N, Huynh DL, Zhang JJ, Kim N, Bak Y, Yoon DY, Yu DY, Lee JC, Gera M, et al: BRM270 inhibits cancer stem cell maintenance via microRNA regulation in chemoresistant A549 lung adenocarcinoma cells. Cell Death Dis 9: 244, 2018.

35. Zhao M, Ang L, Huang J and Wang J: MicroRNAs regulate the epithelial-mesenchymal transition and influence breast cancer invasion and metastasis. Tumour Biol 39: 1010428317691682, 2017.

36. Barbarotto E and Calin GA: MicroRNAs and drug resistance. Drug Resistance Cancer Cells 102: 257-270.

37. Kim J, Yao F, Xiao Z, Sun Y and Ma L: MicroRNAs and metastasis: Small RNAs play big roles. Cancer Metastasis Rev 37: 5-15, 2018 . 
38. McGuire A, Brown JA and Kerin MJ: Metastatic breast cancer: The potential of miRNA for diagnosis and treatment monitoring. Cancer Metastasis Rev 34: 145-155, 2015.

39. Volinia S, Galasso M, Sana ME, Wise TF, Palatini J, Huebner K and Croce CM: Breast cancer signatures for invasiveness and prognosis defined by deep sequencing of microRNA. Proc Natl Acad Sci USA 109: 3024-3029, 2012.

40. Garzon R, Calin GA and Croce CM: MicroRNAs in cancer. Ann Rev Med 60: 167-179, 2009.

41. Klinge CM: Non-coding RNAs in breast cancer: Intracellular and intercellular communication. Noncoding RNA 4: 40, 2018.

42. Kanasty R, Dorkin JR, Vegas A and Anderson D: Delivery materials for siRNA therapeutics. Nat Materials 12: 967-977, 2013.

43. Majumder S and Jacob ST: Emerging role of microRNAs in drug-resistant breast cancer. Gene Expr 15: 141-151, 2011.

44. Papadaki C, Stratigos M, Markakis G, Spiliotaki M, Mastrostamatis G, Nikolaou C, Mavroudis D and Agelaki S: Circulating microRNAs in the early prediction of disease recurrence in primary breast cancer. Breast Cancer Res 20: 72, 2018.

45. Quan Y, Huang X and Quan X: Expression of miRNA-206 and miRNA-145 in breast cancer and correlation with prognosis. Oncol Lett 16: 6638-6642, 2018

46. Wang H, Peng R, Wang J, Qin Z and Xue L: Circulating microRNAs as potential cancer biomarkers: The advantage and disadvantage. Clinical Epigenetics 10: 59, 2018.

47. Abdelrahim M, Safe S, Baker C and Abudayyeh A: RNAi and cancer: Implications and applications. J RNAi Gene Silencing 2: 136-145, 2006.

48. Ewert KK, Zidovska A, Ahmad A, Bouxsein NF, Evans HM, McAllister CS, Samuel CE and Safinya CR: Cationic liposome-nucleic acid complexes for gene delivery and silencing: Pathways and mechanisms for plasmid DNA and siRNA. Top Curr Chem 296: 191-226, 2010.

49. Lin Q, Chen J, Zhang Z and Zheng G: Lipid-based nanoparticles in the systemic delivery of siRNA. Nanomedicine 9: 105-120, 2014.

50. van Zandwijk N, Pavlakis N, Kao SC, Linton A, Boyer MJ, Clarke S, Huynh Y, Chrzanowska A, Fulham MJ, Bailey DL, et al: Safety and activity of microRNA-loaded minicells in patients with recurrent malignant pleural mesothelioma: A first-in-man, phase 1, open-label, dose-escalation study. Lancet Oncol 18: 1386-1396, 2017.

51. Hong DS, Kang YK, Borad M, Sachdev J, Ejadi S, Lim HY, Brenner AJ, Park K, Lee JL, Kim TY, et al: Phase 1 study of MRX34, a liposomal miR-34a mimic, in patients with advanced solid tumours. Br J Cancer 122: 1630-1637, 2020.

52. Anselmo AC and Mitragotri S: Nanoparticles in the clinic: An update. Bioeng Transl Med 4: e10143, 2019.

53. Qattan A: Gene Silencing Agents in Breast Cancer. In: Modulating Gene Expression-Abridging the RNAi and CRISPR-Cas9 Technologies. Singh A and W. Khan M (eds). Intech Open, 2019

54. Stratton MR, Campbell PJ and Futreal PA: The cancer genome. Nature 458: 719-724, 2009.

55. Pedroso de Lima MC, Simões S, Pires P, Faneca H and Düzgüneş N: Cationic lipid-DNA complexes in gene delivery: From biophysics to biological applications. Adv Drug Deliv Rev 47: 277-294, 2001.

56. Meraz IM, Savage DJ, Segura-Ibarra V, Li J, Rhudy J, Gu J and Serda RE: Adjuvant cationic liposomes presenting MPL and IL-12 induce cell death, suppress tumor growth, and alter the cellular phenotype of tumors in a murine model of breast cancer. Mol Pharm 11: 3484-3491, 2014.

57. Yu S, Bi X, Yang L, Wu S, Yu Y, Jiang B, Zhang A, Lan K and Duan S: Co-delivery of paclitaxel and PLK1-targeted siRNA using aptamer-functionalized cationic liposome for synergistic anti-breast cancer effects in vivo. J Biomed Nanotechnol 15 : $1135-1148,2019$.

58. Wang W, Shao A, Zhang N, Fang J, Ruan JJ and Ruan BH: Cationic Polymethacrylate-modified liposomes significantly enhanced doxorubicin delivery and antitumor activity. Sci Rep 7: 43036, 2017.

59. Sun Y, Zhao Y, Zhao X, Lee RJ, Teng L and Zhou C: Enhancing the therapeutic delivery of oligonucleotides by chemical modification and nanoparticle encapsulation. Molecules 22: 1724, 2017.

60. Angelini G, Pisani M, Mobbili G, Marini M and Gasbarri C: Neutral liposomes containing crown ether-lipids as potential DNA vectors. Biochim Biophys Acta 1828: 2506-2512, 2013.

61. Yan Y, Li XQ, Duan JL, Bao CJ, Cui YN, Su ZB, Xu JR, Luo Q, Chen M, Xie Y and Lu WL: Nanosized functional miRNA liposomes and application in the treatment of TNBC by silencing Slug gene. Int J Nanomedicine 14: 3645-3667, 2019.
62. Lu YC, Ou-Yang FU, Hsieh CM, Chang KJ, Chen DR, Tu CW, Wang HC and Hou MF: Pegylated liposomal doxorubicin as adjuvant therapy for stage I-III operable breast cancer. In Vivo 30: 159-163, 2016

63. Xu D, Tian $\mathrm{W}$ and Shen $\mathrm{H}$ : Curcumin prevents induced drug resistance: A novel function? Chin J Cancer Res 23: 218-223, 2011.

64. Tang X, Bi H, Feng $\mathrm{J}$ and Cao J: Effect of curcumin on multidrug resistance in resistant human gastric carcinoma cell line SGC7901/VCR. Acta Pharmacologica Sinica 26: 1009-1016, 2005.

65. Thorn CF, Oshiro C, Marsh S, Hernandez-Boussard T, McLeod H, Klein TE and Altman RB: Doxorubicin pathways: Pharmacodynamics and adverse effects. Pharmacogenet Genomics 21: 440-446, 2011.

66. Zhou S, Li J, Xu H, Zhang S, Chen X, Chen W, Yang S, Zhong S, Zhao J and Tang J: Liposomal curcumin alters chemosensitivity of breast cancer cells to Adriamycin via regulating microRNA expression. Gene 622: 1-12, 2017.

67. Golkar N, Samani SM and Tamaddon AM: Data on cell growth inhibition induced by anti-VEGF siRNA delivered by Stealth liposomes incorporating G2 PAMAM-cholesterol versus Metafectene ${ }^{\circledR}$ as a function of exposure time and siRNA concentration. Data Brief 8: 1018-1023, 2016.

68. Chen J, Sun X, Shao R, Xu Y, Gao J and Liang W: VEGF siRNA delivered by polycation liposome-encapsulated calcium phosphate nanoparticles for tumor angiogenesis inhibition in breast cancer. Int J Nanomedicine 12: 6075-6088, 2017.

69. Leung AK, Tam YY and Cullis PR: Lipid Nanoparticles for short interfering RNA delivery. Adv Genet 88: 71-110, 2014.

70. Fernandez-Piñeiro I, Badiola I and Sanchez A: Nanocarriers for microRNA delivery in cancer medicine. Biotechnol Adv 35: 350-360, 2017.

71. Pandey H, Rani R and Agarwal V: Liposome and their applications in cancer therapy. Brazilian Arch Biol Technol: 59, 2016 http://dx.doi.org/10.1590/1678-4324-2016150477.

72. Liu HM, Zhang YF, Xie YD, Cai YF, Li BY, Li W, Zeng LY, Li YL and Yu RT: Hypoxia-responsive ionizable liposome delivery siRNA for glioma therapy. Int J Nanomedicine 12: 1065-1083, 2017

73. Modi S, Xiang TX and Anderson BD: Enhanced active liposomal loading of a poorly soluble ionizable drug using supersaturated drug solutions. J Control Release 162: 330-339, 2012.

74. Fenske DB, Chonn A and Cullis PR: Liposomal nanomedicines: An emerging field. Toxicol Pathol 36: 21-29, 2008.

75. Johnston MJ, Semple SC, Klimuk SK, Edwards K, Eisenhardt ML, Leng EC, Karlsson G, Yanko D and Cullis PR: Therapeutically optimized rates of drug release can be achieved by varying the drug-to-lipid ratio in liposomal vincristine formulations. Biochim Biophys Acta 1758: 55-64, 2006.

76. Lujan H, Griffin WC, Taube JH and Sayes CM: Synthesis and characterization of nanometer-sized liposomes for encapsulation and microRNA transfer to breast cancer cells. Int J Nanomedicine 14: 5159-5173, 2019.

77. de Antonellis P, Liguori L, Falanga A, Carotenuto $M$, Ferrucci V, Andolfo I, Marinaro F, Scognamiglio I, Virgilio A, De Rosa G, et al: MicroRNA 199b-5p delivery through stable nucleic acid lipid particles (SNALPs) in tumorigenic cell lines. Naunyn Schmiedebergs Arch Pharmacol 386: 287-302, 2013.

78. Samanta S, Rajasingh S, Drosos N, Zhou Z, Dawn B and Rajasingh J: Exosomes: New molecular targets of diseases. Acta pharmacologica Sinica 39: 501-513, 2018.

79. Bhome R, Del Vecchio F, Lee GH, Bullock MD, Primrose JN, Sayan AE and Mirnezami AH: Exosomal microRNAs (exomiRs): Small molecules with a big role in cancer. Cancer Lett 420: 228-235, 2018

80. Zhang J, Li S, Li L, Li M, Guo C, Yao J and Mi S: Exosome and exosomal microRNA: Trafficking, sorting, and function. Genomics Proteomics Bioinformatics 13: 17-24, 2015.

81. Xin Y, Wang X, Meng K, Ni C, Lv Z and Guan D: Identification of exosomal miR-455-5p and miR-1255a as therapeutic targets for breast cancer. Biosci Rep 40: BSR20190303, 2020.

82. Harris DA, Patel SH, Gucek M, Hendrix A, Westbroek W and Taraska JW: Exosomes released from breast cancer carcinomas stimulate cell movement. PLoS One 10: e0117495, 2015.

83. Lin R, Wang $\mathrm{S}$ and Zhao RC: Exosomes from human adipose-derived mesenchymal stem cells promote migration through Wnt signaling pathway in a breast cancer cell model. Mol Cell Biochem 383: 13-20, 2013.

84. Wu CY, Du SL, Zhang J, Liang AL and Liu YJ: Exosomes and breast cancer: A comprehensive review of novel therapeutic strategies from diagnosis to treatment. Cancer Gene Ther 24: 6-12, 2017. 
85. Taylor DD and Gercel-Taylor C: MicroRNA signatures of tumor-derived exosomes as diagnostic biomarkers of ovarian cancer. Gynecol Oncol 110: 13-21, 2008.

86. Silva J, Garcia V, Zaballos A, Provencio M, Lombardía L, Almonacid L, García JM, Domínguez G, Peña C, Diaz R, et al: Vesicle-related microRNAs in plasma of nonsmall cell lung cancer patients and correlation with survival. Eur Respir J 37: 617-623, 2011

87. Rabinowits G, Gerçel-Taylor C, Day JM, Taylor DD and Kloecker GH: Exosomal MicroRNA: A diagnostic marker for lung cancer. Clinical Lung Cancer 10: 42-46, 2009.

88. Wu H, Wang Q, Zhong H, Li L, Zhang Q, Huang Q and Yu Z: Differentially expressed microRNAs in exosomes of patients with breast cancer revealed by next-generation sequencing. Oncol Rep 43: 240-250, 2019.

89. Allen TM, Hansen C, Martin F, Redemann C and Yau-Young A: Liposomes containing synthetic lipid derivatives of poly(ethylene glycol) show prolonged circulation half-lives in vivo. Biochim Biophys Acta 1066: 29-36, 1991.

90. Klibanov AL, Maruyama K, Torchilin VP and Huang L: Amphipathic polyethyleneglycols effectively prolong the circulation time of liposomes. FEBS Lett 268: 235-237, 1990 .

91. Senior J, Delgado C, Fisher D, Tilcock C and Gregoriadis G: Influence of surface hydrophilicity of liposomes on their interaction with plasma protein and clearance from the circulation: Studies with poly(ethylene glycol)-coated vesicles. Biochim Biophys Acta 1062: 77-82, 1991.

92.Immordino ML, Dosio F and Cattel L: Stealth liposomes: Review of the basic science, rationale, and clinical applications, existing and potential. Int J Nanomedicine 1: 297-315, 2006

93. Caliceti P: Pharmacokinetic and biodistribution properties of poly(ethylene glycol)-protein conjugates. Adv Drug Delivery Rev 55: 1261-1277, 2003.

94. Barenholz Y: Doxil ${ }^{\circledR}$ The first FDA-approved Nano-drug: Lessons learned. J Control Release 160: 117-134, 2012.

95. Vakhshiteh F, Khabazian E, Atyabi F, Ostad SN, Madjd Z and Dinarvand R: Peptide-conjugated liposomes for targeted miR-34a delivery to suppress breast cancer and cancer stem-like population. J Drug Deliv Sci Technol 57: 101687, 2020.

96. Amstad E, Kohlbrecher J, Müller E, Schweizer T, Textor M and Reimhult E: Triggered release from liposomes through magnetic actuation of iron oxide nanoparticle containing membranes. Nano Lett 11: 1664-1670, 2011.
97. Nahire R, Hossain R, Patel R, Paul S, Meghnani V, Ambre AH, Gange KN, Katti KS, Leclerc E, Srivastava DK, et al: pH-Triggered echogenicity and contents release from liposomes. Mol Pharm 11: 4059-4068, 2014.

98. Khan DR, Webb MN, Cadotte TH and Gavette MN: Use of targeted liposome-based chemotherapeutics to treat breast cancer. Breast Cancer (Auckl) 9 (Suppl 2): S1-S5, 2015.

99. Ta T and Porter TM: Thermosensitive liposomes for localized delivery and triggered release of chemotherapy. J Control Release 169: 112-125, 2013.

100. Elegbede AI, Banerjee J, Hanson AJ, Tobwala S, Ganguli B, Wang R, Lu X, Srivastava DK and Mallik S: Mechanistic studies of the triggered release of liposomal contents by matrix metalloproteinase-9. J Am Chem Soc 130: 10633-10642, 2008.

101. Brown S and Khan DR: The treatment of breast cancer using liposome technology. J Drug Deliv 2012: 212965, 2012.

102. Sneider A, Jadia R, Piel B, VanDyke D, Tsiros C and Rai P. Engineering remotely triggered liposomes to target triple negative breast cancer. Oncomedicine 2: 1-13, 2017.

103. Yang G, Liu Z, Li Y, Hou Y, Fei X, Su C, Wang S, Zhuang Z and Guo Z: Facile synthesis of black phosphorus-Au nanocomposites for enhanced photothermal cancer therapy and surface-enhanced Raman scattering analysis. Biomater Sci 5: 2048-2055, 2017.

104. Qiu M, Ren WX, Jeong T, Won M, Park GY, Sang DK, Liu LP, Zhang $\mathrm{H}$ and Kim JS: Omnipotent phosphorene: A next-generation, two-dimensional nanoplatform for multidisciplinary biomedical applications. Chem Soc Rev 47: 5588-5601, 2018.

105. Sun S, Xu Y, Fu P, Chen M, Sun S, Zhao R, Wang J, Liang X and Wang S: Ultrasound-targeted photodynamic and gene dual therapy for effectively inhibiting triple negative breast cancer by cationic porphyrin lipid microbubbles loaded with HIF1 $\alpha$-siRNA. Nanoscale 10: 19945-19956, 2018.

106. Tang J, Li B, Howard CB, Mahler SM, Thurecht KJ, Wu Y, Huang L and Xu ZP: Multifunctional lipid-coated calcium phosphate nanoplatforms for complete inhibition of large triple negative breast cancer via targeted combined therapy. Biomaterials 216: 119232, 2019.

107. Ghasemiyeh P and Mohammadi-Samani S: Solid lipid nanoparticles and nanostructured lipid carriers as novel drug delivery systems: Applications, advantages and disadvantages. Res Pharma Sci 13: 288-303, 2018 